\title{
<Supporting Information>
}

\section{Size and Shape Selectivity of Host Networks Built \\ Based on Tunable Secondary Building Units}

\author{
Dohyun Moon and Myoung Soo Lah* \\ Department of Chemistry and Applied Chemistry, College of Science and Technology, \\ Hanyang University, Ansan, Kyunggi-Do 426-791, Korea \\ tel:( +82)- 31-400-5496 \\ fax: (+82)- 31-407-3863 \\ E-mail: mslah@hanyang.ac.kr
}




\section{Crystal structure analysis}

All structures were solved by direct methods and refined by full-matrix least-squares calculations with SHELXTL-PULS software package (Ver. 5.1). ${ }^{1}$

$\left[\mathbf{M n}_{\mathbf{6}}(\mathbf{p s h z})_{6}(\boldsymbol{d m f})_{2}(\mathbf{b p e a})_{2}\right], 2$ : Several non-coordinating solvent sites $^{2}$ were observed in the channel area. However, all of them were severely disordered or partly resolved. Refinement of the structure without disordered or partly resolved $d m f$ molecules converged at final $\mathrm{R} 1=0.1315$ for 9570 reflections of $\mathrm{I}>2 \sigma(\mathrm{I}), \mathrm{R} 1=0.1470, \mathrm{wR} 2=0.4447$ for all 11471 reflections. Largest difference peak and hole 3.002 and $-0.703 \mathrm{e} \cdot \AA^{-3}$ respectively. Structure refinement of the network following modification of the data for the disordered electron density with the SQUEEZE routine led to better refinement. ${ }^{2}$ This refinement converged at final $\mathrm{R} 1=0.0586$ for 9854 reflections of $\mathrm{I}>2 \sigma(\mathrm{I}), \mathrm{R} 1=0.0671, \mathrm{wR} 2=0.1576$ for all 11471 reflections. Largest difference peak and hole 0.975 and $-0.497 \mathrm{e} \cdot \AA^{-3}$ respectively.

$\left[\mathbf{M n}_{6}(\mathbf{h s h z})_{6}(\boldsymbol{d m f})_{2}(\text { bpea })_{2}\right]$, 3: Several non-coordinating solvent sites $^{2}$ were observed in the channel area. However, all of them were severely disordered or partly resolved. Refinement of the structure without disordered or partly resolved $d m f$ molecules converged at final $\mathrm{R} 1=0.1389$ for 9277 reflections of $\mathrm{I}>2 \sigma(\mathrm{I}), \mathrm{R} 1=0.1594, \mathrm{wR} 2=0.4447$ for all 11578 reflections. Largest difference peak and hole 3.801 and $-0.754 \mathrm{e} \cdot \AA^{-3}$ respectively. Structure refinement of the network following modification of the data for the disordered electron density with the SQUEEZE routine led to better refinement. ${ }^{2}$ This refinement converged at final $\mathrm{R} 1=0.0583$ for 9671 reflections of $\mathrm{I}>2 \sigma(\mathrm{I}), \mathrm{R} 1=0.0706, \mathrm{wR} 2=0.1564$ for all 11578 reflections. Largest difference peak and hole 0.482 and $-0.427 \mathrm{e} \cdot \AA^{-3}$ respectively.

$\left[\mathbf{M n}_{\mathbf{6}}(\mathbf{a s h z})_{6}(\mathbf{b p e a})_{2}(\boldsymbol{d m f})_{2}\right] \cdot \mathbf{x}(\boldsymbol{m b z}) \cdot \mathbf{y}(\boldsymbol{d} \boldsymbol{m f}), \mathbf{4}$ : Several $m b z$ and/or $d m f$ sites were observed in the channel area. However, all of them were severely disordered or partly resolved. Refinement of the structure without disordered or partly resolved electron density 
converged at final $\mathrm{R} 1=0.1482$ for 6606 reflections of $\mathrm{I}>2 \sigma(\mathrm{I}), \mathrm{R} 1=0.2707, \mathrm{wR} 2=0.4521$ for all 17189 reflections. Largest difference peak and hole 1.812 and $-0.656 \mathrm{e} \cdot \AA^{-3}$ respectively. Structure refinement of the network following modification of the data for the disordered electron density with the SQUEEZE routine led to better refinement. ${ }^{2}$ This refinement converged at final $\mathrm{R} 1=0.0507$ for 6578 reflections of $\mathrm{I}>2 \sigma(\mathrm{I}), \mathrm{R} 1=0.1366$, $\mathrm{wR} 2=0.1208$ for all 11471 reflections. Largest difference peak and hole 0.260 and -0.238 e. $\AA^{-3}$ respectively.

$\left[\mathbf{M n}_{6}(\mathbf{a s h z})_{6}(\mathbf{b p e a})_{2}(\boldsymbol{d m f})_{2}\right] \cdot \mathbf{x}(\boldsymbol{d e p}) \cdot \mathbf{y}(\boldsymbol{d m f}), \mathbf{1 0}$ : A half site of a dep was observed in the minor channel area. Several other dep and/or $d m f$ sites were also observed in the major channel area. Refinement of the structure without disordered or partly resolved electron density converged at final $\mathrm{R} 1=0.1094$ for 12237 reflections of $\mathrm{I}>2 \sigma(\mathrm{I}), \mathrm{R} 1=0.1452$, wR2 $=0.3600$ for all 17581 reflections. Largest difference peak and hole 1.981 and $-0.617 \mathrm{e} \cdot \AA^{-3}$ respectively. Structure refinement of the network following modification of the data for the disordered electron density with the SQUEEZE routine led to better refinement. ${ }^{2}$ This refinement converged at final $\mathrm{R} 1=0.0788$ for 12182 reflections of $\mathrm{I}>2 \sigma(\mathrm{I}), \mathrm{R} 1=0.1065$, $\mathrm{wR} 2=0.2495$ for all 17581 reflections. Largest difference peak and hole 1.402 and -0.565 $\mathrm{e} \cdot \AA^{-3}$ respectively.

$\left[\mathbf{M n}_{\mathbf{6}}(\mathbf{a s h z})_{\mathbf{6}}(\mathbf{b p e a})_{2}(\boldsymbol{d m f})_{2}\right] \cdot \mathbf{x}(\boldsymbol{d p p}) \cdot \mathbf{y}(\boldsymbol{d m f}), \mathbf{1 1}$ : Several $d p p$ and/or $d m f$ sites were observed in the channel area. However, all of them were severely disordered or partly resolved. Refinement of the structure without disordered or partly resolved electron density converged at final $\mathrm{R} 1=0.1482$ for 6441 reflections of $\mathrm{I}>2 \sigma(\mathrm{I}), \mathrm{R} 1=0.2616$, wR2 $=0.4298$ for all 17350 reflections. Largest difference peak and hole 1.795 and $-0.711 \mathrm{e} \cdot \AA^{-3}$ respectively. Structure refinement of the network following modification of the data for the disordered electron density with the SQUEEZE routine led to better refinement. ${ }^{2}$ This refinement converged at final $\mathrm{R} 1=0.0437$ for 6453 reflections of $\mathrm{I}>2 \sigma(\mathrm{I}), \mathrm{R} 1=0.1342$, 
$\mathrm{wR} 2=0.0971$ for all 17350 reflections. Largest difference peak and hole 0.271 and -0.182 e. $\AA^{-3}$ respectively.

$\left[\mathrm{Mn}_{6}(\mathbf{p s h z})_{6}(\mathrm{bpea})_{2}(\boldsymbol{d m f})_{2}\right] \cdot(\mathrm{ibp}) \cdot \mathbf{y}(\boldsymbol{d m f}), \mathbf{1 4}$ : A half site of a $i b p$ was observed in the minor channel area and a $d m f$ site with partial occupancy was observed in the major channel area. Several other partly resolved $i b p$ and/or $d m f$ sites were also observed in the major channel area. Refinement of the structure without disordered or partly resolved electron density converged at final $\mathrm{R} 1=0.1042$ for 10014 reflections of $\mathrm{I}>2 \sigma(\mathrm{I}), \mathrm{R} 1=0.1679$, wR2 $=0.3620$ for all 17690 reflections. Largest difference peak and hole 1.632 and $-0.551 \mathrm{e} \cdot \AA^{-3}$ respectively. Structure refinement of the network following modification of the data for the disordered electron density with the SQUEEZE routine led to better refinement. ${ }^{2}$ This refinement converged at final $\mathrm{R} 1=0.0832$ for 10104 reflections of $\mathrm{I}>2 \sigma(\mathrm{I}), \mathrm{R} 1=0.1254$, $w R 2=0.2629$ for all 17690 reflections. Largest difference peak and hole 1.133 and -0.448 $\mathrm{e} \cdot \AA^{-3}$ respectively.

$\left[\mathrm{Mn}_{6}(\mathbf{p s h z})_{6}(\mathbf{b p e a})_{2}(\boldsymbol{d m f})_{2}\right] \cdot(e b z) \cdot \mathbf{y}(\boldsymbol{d m f}), \mathbf{1 5}$ : A half site of a $e b z$ was observed in the minor channel area and a disordered $\operatorname{dmf}$ site was observed in the major channel area. Several other partly resolved $e b z$ and/or $d m f$ sites were also observed in the major channel area. Refinement of the structure without disordered or partly resolved electron density converged at final $\mathrm{R} 1=0.1443$ for 10933 reflections of $\mathrm{I}>2 \sigma(\mathrm{I}), \mathrm{R} 1=0.2013, \mathrm{wR} 2=0.4286$ for all 17487 reflections. Largest difference peak and hole 1.712 and $-0.690 \mathrm{e} \cdot \AA^{-3}$ respectively. Structure refinement of the network following modification of the data for the disordered electron density with the SQUEEZE routine led to better refinement. ${ }^{2}$ This refinement converged at final $\mathrm{R} 1=0.0946$ for 10648 reflections of $\mathrm{I}>2 \sigma(\mathrm{I}), \mathrm{R} 1=0.1347, \mathrm{wR} 2=$ 0.2931 for all 17487 reflections. Largest difference peak and hole 1.112 and $-0.573 \mathrm{e} \cdot \AA^{-3}$ respectively. 
$\left[\mathbf{M n}_{6}(\mathbf{p s h z})_{6}(\mathbf{b p e a})_{2}(\boldsymbol{d m f})_{2}\right] \cdot(\mathbf{p b z}) \cdot \mathbf{y}(\boldsymbol{d m f}), \mathbf{1 6}$ : A $d m f$ site was observed in the interconnection area between the major and minor channels. Several other partly resolved $p b z$ and/or $d m f$ sites were also observed in the major channel area. Refinement of the structure without disordered or partly resolved electron density converged at final R $1=0.1419$ for 7939 reflections of $\mathrm{I}>2 \sigma(\mathrm{I}), \mathrm{R} 1=0.2513, \mathrm{wR} 2=0.4282$ for all 17375 reflections. Largest difference peak and hole 1.665 and $-0.827 \mathrm{e} \cdot \AA^{-3}$ respectively. Structure refinement of the network following modification of the data for the disordered electron density with the SQUEEZE routine led to better refinement. ${ }^{2}$ This refinement converged at final $\mathrm{R} 1=0.0914$ for 7896 reflections of $\mathrm{I}>2 \sigma(\mathrm{I}), \mathrm{R} 1=0.1737, \mathrm{wR} 2=0.2476$ for all 17690 reflections. Largest difference peak and hole 0.897 and $-0.704 \mathrm{e} \cdot \AA^{-3}$ respectively.

$\left[\mathbf{M n}_{6}(\mathbf{p s h z})_{6}(\mathbf{b p e a})_{2}(\boldsymbol{d m f})_{2}\right] \cdot(\boldsymbol{b b z}) \cdot \mathbf{y}(\boldsymbol{d m f}), \mathbf{1 7}$ : Two $d m f$ sites were observed in the major channels. Several other partly resolved $b b z$ and/or $d m f$ sites were also observed in the channel area. Refinement of the structure without disordered or partly resolved electron density converged at final $\mathrm{R} 1=0.1025$ for 9982 reflections of $\mathrm{I}>2 \sigma(\mathrm{I}), \mathrm{R} 1=0.1669, \mathrm{wR} 2=0.3752$ for all 17696 reflections. Largest difference peak and hole 1.759 and $-0.743 \mathrm{e} \cdot \AA^{-3}$ respectively. Structure refinement of the network following modification of the data for the disordered electron density with the SQUEEZE routine led to better refinement. ${ }^{2}$ This refinement converged at final $\mathrm{R} 1=0.0709$ for 10127 reflections of $\mathrm{I}>2 \sigma(\mathrm{I}), \mathrm{R} 1=0.1089$, $\mathrm{wR} 2=0.2255$ for all 17696 reflections. Largest difference peak and hole 1.229 and -0.480 $\mathrm{e} \cdot \AA^{-3}$ respectively.

$\left[\mathbf{M n}_{6}(\mathbf{p s h z})_{6}(\mathbf{b p e a})_{2}(\boldsymbol{d m f})_{2}\right] \cdot(\boldsymbol{d m p}) \cdot \mathbf{y}(\boldsymbol{d m f}), \mathbf{1 8}$ : A half site of a $d m p$ was observed in the minor channel area. Several other partly resolved $d m p$ and/or $d m f$ sites were also observed in the major channel area. Refinement of the structure without disordered or partly resolved electron density converged at final $\mathrm{R} 1=0.1063$ for 9011 reflections of $\mathrm{I}>2 \sigma(\mathrm{I}), \mathrm{R} 1=$ $0.1809, \mathrm{wR} 2=0.3787$ for all 17712 reflections. Largest difference peak and hole 1.302 and - 
$0.749 \mathrm{e} \cdot \AA^{-3}$ respectively. Structure refinement of the network following modification of the data for the disordered electron density with the SQUEEZE routine led to better refinement. ${ }^{2}$ This refinement converged at final $\mathrm{R} 1=0.0879$ for 9140 reflections of $\mathrm{I}>2 \sigma(\mathrm{I}), \mathrm{R} 1=$ $0.1334, \mathrm{wR} 2=0.2855$ for all 17712 reflections. Largest difference peak and hole 1.247 and $0.734 \mathrm{e} \cdot \AA^{-3}$ respectively.

$\left[\mathbf{M n}_{6}(\mathbf{p s h z})_{6}(\mathbf{b p e a})_{2}(\boldsymbol{d m f})_{2}\right] \cdot(\boldsymbol{d e p}) \cdot \mathbf{y}(\boldsymbol{d m f}), \mathbf{1 9}$ : A half site of a dep was observed in the minor channel area. Several other partly resolved $d m p$ and/or $d m f$ sites were also observed in the major channel area. Refinement of the structure without disordered or partly resolved electron density converged at final $\mathrm{R} 1=0.1127$ for 12645 reflections of $\mathrm{I}>2 \sigma(\mathrm{I}), \mathrm{R} 1=$ $0.1509, \mathrm{wR} 2=0.3914$ for all 17712 reflections. Largest difference peak and hole 2.134 and $0.811 \mathrm{e} \cdot \AA^{-3}$ respectively. Structure refinement of the network following modification of the data for the disordered electron density with the SQUEEZE routine led to better refinement. ${ }^{2}$ This refinement converged at final $\mathrm{R} 1=0.0883$ for 13389 reflections of $\mathrm{I}>2 \sigma(\mathrm{I}), \mathrm{R} 1=$ $0.1100, \mathrm{wR} 2=0.2710$ for all 17712 reflections. Largest difference peak and hole 1.641 and $0.616 \mathrm{e} \cdot \AA^{-3}$ respectively.

$\left[\mathrm{Mn}_{6}(\mathbf{p s h z})_{6}(\mathrm{bpea})_{2}(\boldsymbol{d m f})_{2}\right] \cdot(\boldsymbol{d p p}) \cdot \mathbf{y}(\boldsymbol{d m f}), \mathbf{2 0}$ : A $d m f$ site was observed in the interconnection area between the major and minor channels. Several other partly resolved $d p p$ and/or $d m f$ sites were also observed in the major channel area. Refinement of the structure without disordered or partly resolved electron density converged at final $\mathrm{R} 1=0.1503$ for 6796 reflections of $\mathrm{I}>2 \sigma(\mathrm{I}), \mathrm{R} 1=0.2824, \mathrm{wR} 2=0.4511$ for all 17506 reflections. Largest difference peak and hole 1.748 and $-0.834 \mathrm{e} \cdot \AA^{-3}$ respectively. Structure refinement of the network following modification of the data for the disordered electron density with the SQUEEZE routine led to better refinement. ${ }^{2}$ This refinement converged at final R1 $=0.0946$ for 6838 reflections of $\mathrm{I}>2 \sigma(\mathrm{I}), \mathrm{R} 1=0.1984, \mathrm{wR} 2=0.2736$ for all 17712 reflections. Largest difference peak and hole 0.707 and $-0.657 \mathrm{e} \cdot \AA^{-3}$ respectively. 
$\left[\mathbf{M n}_{6}(\mathbf{p s h z})_{6}(\mathbf{b p e a})_{2}(\boldsymbol{d m f})_{2}\right] \cdot(\boldsymbol{d b p}) \cdot \mathbf{y}(\boldsymbol{d m f}), 21$ : Several partly resolved or disorderd $d b p$ and/or $d m f$ sites were observed in the channel area. Refinement of the structure without disordered or partly resolved electron density converged at final R1 $=0.1259$ for 5603 reflections of $\mathrm{I}>2 \sigma(\mathrm{I}), \mathrm{R} 1=0.2863, \mathrm{wR} 2=0.4122$ for all 17465 reflections. Largest difference peak and hole 1.718 and $-0.602 \mathrm{e} \cdot \AA^{-3}$ respectively. Structure refinement of the network following modification of the data for the disordered electron density with the SQUEEZE routine led to better refinement. ${ }^{2}$ This refinement converged at final $\mathrm{R} 1=0.0612$ for 5598 reflections of $\mathrm{I}>2 \sigma(\mathrm{I}), \mathrm{R} 1=0.1593, \mathrm{wR} 2=0.1573$ for all 17465 reflections. Largest difference peak and hole 0.643 and $-0.358 \mathrm{e} \cdot \AA^{-3}$ respectively.

$\left[\mathbf{M n}_{6}(\mathbf{h s h z})_{6}(\mathbf{b p e a})_{2}(\boldsymbol{d m f})_{2}\right] \cdot(\boldsymbol{m b z}) \cdot \mathbf{y}(\boldsymbol{d m f}), \mathbf{2 2}$ : A half site of a $m b z$ was observed in the minor channel area. Several other $m b z$ and/or $d m f$ sites were also observed in the major channel area. Refinement of the structure without disordered or partly resolved electron density converged at final $\mathrm{R} 1=0.1131$ for 9604 reflections of $\mathrm{I}>2 \sigma(\mathrm{I}), \mathrm{R} 1=0.1934$, wR2 $=$ 0.3847 for all 17702 reflections. Largest difference peak and hole 1.977 and $-0.955 \mathrm{e} \cdot \AA^{-3}$ respectively. Structure refinement of the network following modification of the data for the disordered electron density with the SQUEEZE routine led to better refinement. ${ }^{2}$ This refinement converged at final $\mathrm{R} 1=0.0881$ for 9978 reflections of $\mathrm{I}>2 \sigma(\mathrm{I}), \mathrm{R} 1=0.1407$, $w R 2=0.2622$ for all 17702 reflections. Largest difference peak and hole 1.107 and -0.533 e. $\AA^{-3}$ respectively.

$\left[\mathrm{Mn}_{6}(\mathbf{h s h z})_{6}(\mathbf{b p e a})_{2}(\boldsymbol{d m f})_{2}\right] \cdot(\boldsymbol{e b z}) \cdot \mathbf{y}(\boldsymbol{d m f}), 24$ : A half site of a $e b z$ was observed in the minor channel area. Several other $m b z$ and/or $d m f$ sites were also observed in the major channel area. Refinement of the structure without disordered or partly resolved electron density converged at final $\mathrm{R} 1=0.0980$ for 9167 reflections of $\mathrm{I}>2 \sigma(\mathrm{I}), \mathrm{R} 1=0.1752$, wR2 $=$ 0.3605 for all 17656 reflections. Largest difference peak and hole 1.985 and $-0.779 \mathrm{e} \cdot \AA^{-3}$ respectively. Structure refinement of the network following modification of the data for the 
disordered electron density with the SQUEEZE routine led to better refinement. ${ }^{2}$ This refinement converged at final $\mathrm{R} 1=0.0551$ for 9297 reflections of $\mathrm{I}>2 \sigma(\mathrm{I}), \mathrm{R} 1=0.1054$, $w \mathrm{R} 2=0.1534$ for all 17656 reflections. Largest difference peak and hole 0.549 and -0.442 $\mathrm{e} \cdot \AA^{-3}$ respectively.

$\left[\mathbf{M n}_{6}(\mathbf{h s h z})_{6}(\mathbf{b p e a})_{2}(\boldsymbol{d m f})_{2}\right] \cdot(\mathbf{p b z}) \cdot \mathbf{y}(\boldsymbol{d m f}), 25:$ A $d m f$ site was observed in the major channels. Several other partly resolved $\mathrm{pbz}$ and/or $d m f$ sites were also observed in the channel area. Refinement of the structure without disordered or partly resolved electron density converged at final $\mathrm{R} 1=0.1109$ for 6802 reflections of $\mathrm{I}>2 \sigma(\mathrm{I}), \mathrm{R} 1=0.2371, \mathrm{wR} 2=0.3750$ for all 17633 reflections. Largest difference peak and hole 1.597 and $-0.587 \mathrm{e} \cdot \AA^{-3}$ respectively. Structure refinement of the network following modification of the data for the disordered electron density with the SQUEEZE routine led to better refinement. ${ }^{2}$ This refinement converged at final $\mathrm{R} 1=0.0680$ for 6874 reflections of $\mathrm{I}>2 \sigma(\mathrm{I}), \mathrm{R} 1=0.1700$, $\mathrm{wR} 2=0.1722$ for all 17633 reflections. Largest difference peak and hole 0.489 and -0.273 $\mathrm{e} \cdot \AA^{-3}$ respectively.

$\left[\mathrm{Mn}_{6}(\mathbf{h s h z})_{6}(\mathbf{b p e a})_{2}(\boldsymbol{d m f})_{2}\right] \cdot(\boldsymbol{b b z}) \cdot \mathbf{y}(\boldsymbol{d m f}), \mathbf{2 6}$ : A $d m f$ site with partial occupancy was observed in the major channels. Several other partly resolved $b b z$ and/or $d m f$ sites were also observed in the channel area. Refinement of the structure without disordered or partly resolved electron density converged at final $\mathrm{R} 1=0.1058$ for 6656 reflections of $\mathrm{I}>2 \sigma(\mathrm{I}), \mathrm{R} 1$ $=0.2400, \mathrm{wR} 2=0.3690$ for all 17627 reflections. Largest difference peak and hole 1.771 and $-0.490 \mathrm{e} \cdot \AA^{-3}$ respectively. Structure refinement of the network following modification of the data for the disordered electron density with the SQUEEZE routine led to better refinement. ${ }^{2}$ This refinement converged at final $\mathrm{R} 1=0.0680$ for 6785 reflections of $\mathrm{I}>2 \sigma(\mathrm{I}), \mathrm{R} 1=$ 0.1786, wR2 $=0.1684$ for all 17633 reflections. Largest difference peak and hole 0.508 and $0.316 \mathrm{e} \cdot \AA^{-3}$ respectively. 
$\left[\mathbf{M n}_{6}(\mathbf{h s h z})_{6}(\mathbf{b p e a})_{2}(\boldsymbol{d m f})_{2}\right] \cdot(\boldsymbol{d e p}) \cdot \mathbf{y}(\boldsymbol{d m f}), \mathbf{2 8}$ : A $d m f$ site with partial occupancy was observed in the minor channels. Several other partly resolved $\operatorname{dep}$ and/or $d m f$ sites were also observed in the other channel area. Refinement of the structure without disordered or partly resolved electron density converged at final $\mathrm{R} 1=0.1268$ for 8368 reflections of $\mathrm{I}>2 \sigma(\mathrm{I}), \mathrm{R} 1$ $=0.2257, \mathrm{wR} 2=0.4236$ for all 17366 reflections. Largest difference peak and hole 2.006 and $-0.590 \mathrm{e} \cdot \AA^{-3}$ respectively. Structure refinement of the network following modification of the data for the disordered electron density with the SQUEEZE routine led to better refinement. ${ }^{2}$ This refinement converged at final $\mathrm{R} 1=0.1001$ for 8866 reflections of $\mathrm{I}>2 \sigma(\mathrm{I}), \mathrm{R} 1=$ $0.1612, w R 2=0.3045$ for all 17366 reflections. Largest difference peak and hole 1.194 and $0.437 \mathrm{e} \cdot \AA^{-3}$ respectively.

$\left[\mathbf{M n}_{6}(\mathbf{h s h z})_{6}(\mathbf{b p e a})_{2}(\boldsymbol{d m f})_{2}\right] \cdot(\boldsymbol{d p p}) \cdot \mathbf{y}(\boldsymbol{d m f}), \mathbf{2 9}$ : Several partly resolved $d p p$ and/or $d m f$ sites were also observed in the channel area. Refinement of the structure without disordered or partly resolved electron density converged at final $\mathrm{R} 1=0.1181$ for 7287 reflections of $\mathrm{I}>$ $2 \sigma(\mathrm{I}), \mathrm{R} 1=0.2262, \mathrm{wR} 2=0.3906$ for all 17323 reflections. Largest difference peak and hole 2.084 and $-0.558 \mathrm{e} \cdot \AA^{-3}$ respectively. Structure refinement of the network following modification of the data for the disordered electron density with the SQUEEZE routine led to better refinement. ${ }^{2}$ This refinement converged at final $\mathrm{R} 1=0.0651$ for 7427 reflections of $\mathrm{I}>$ $2 \sigma(\mathrm{I}), \mathrm{R} 1=0.1553, \mathrm{wR} 2=0.1546$ for all 17323 reflections. Largest difference peak and hole 0.569 and $-0.243 \mathrm{e} \cdot \AA^{-3}$ respectively.

$\left[\mathbf{M n}_{6}(\mathbf{h s h z})_{6}(\mathbf{b p e a})_{2}(\boldsymbol{d m f})_{2}\right] \cdot(\boldsymbol{d b p}) \cdot \mathbf{y}(\boldsymbol{d m f}), \mathbf{3 0}$ : A $d m f$ site with partial occupancy was observed in the minor channels. Several other partly resolved $d b p$ and/or $d m f$ sites were also observed in the other channel area. Refinement of the structure without disordered or partly resolved electron density converged at final $\mathrm{R} 1=0.1200$ for 7873 reflections of $\mathrm{I}>2 \sigma(\mathrm{I}), \mathrm{R} 1$ $=0.2185, \mathrm{wR} 2=0.3880$ for all 17802 reflections. Largest difference peak and hole 2.500 and $-0.588 \mathrm{e} \cdot \AA^{-3}$ respectively. Structure refinement of the network following modification of the 
data for the disordered electron density with the SQUEEZE routine led to better refinement. ${ }^{2}$ This refinement converged at final $\mathrm{R} 1=0.0605$ for 7884 reflections of $\mathrm{I}>2 \sigma(\mathrm{I}), \mathrm{R} 1=$ $0.1402, \mathrm{wR} 2=0.1413$ for all 17802 reflections. Largest difference peak and hole 0.466 and $0.299 \mathrm{e} \cdot \AA^{-3}$ respectively.

\section{References}

(1) Sheldrick, G. M. SHELXTL-PLUS. Crystal Structure Analysis Package; Bruker Analytical X-ray, Madison, WI, 1997.

(2) Platon program: Spek, A. L. Acta Crystallogr. Sect. A 1990, 46, 194-201. 

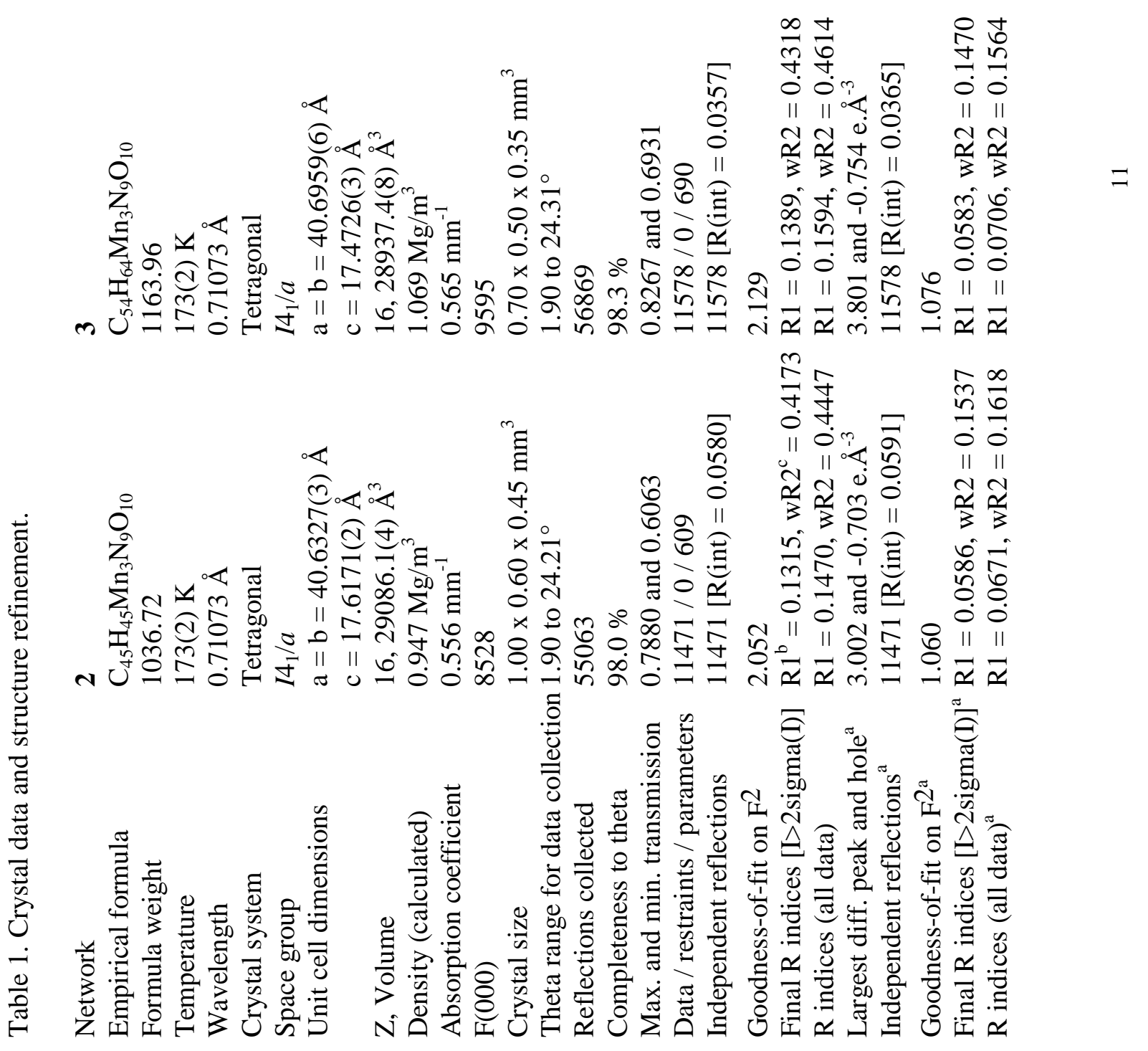


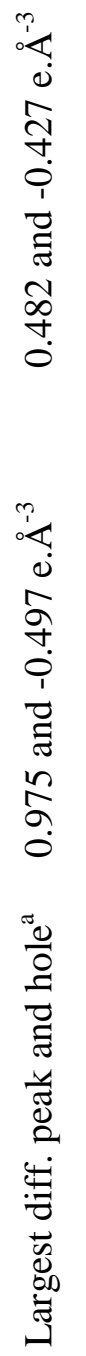



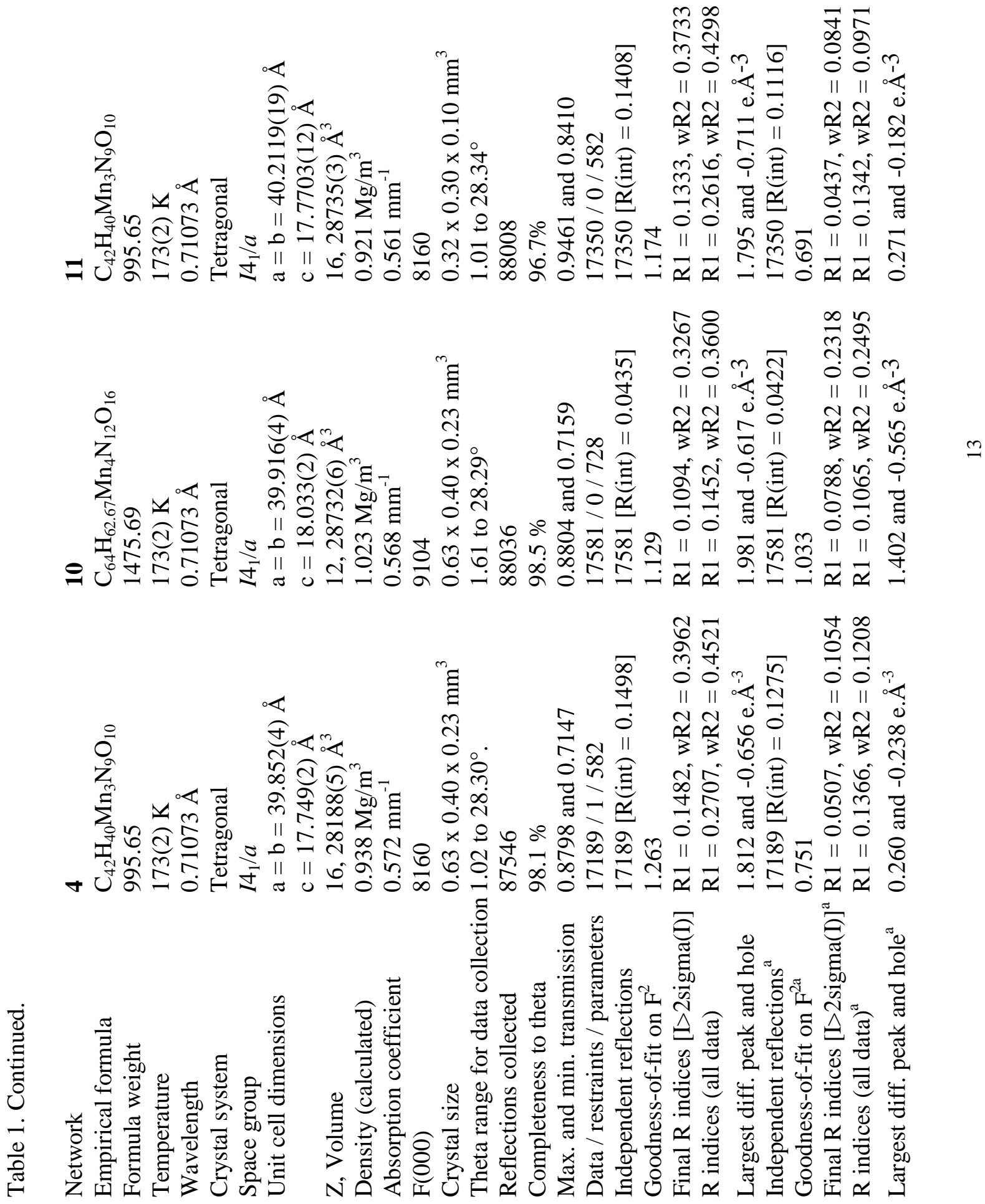

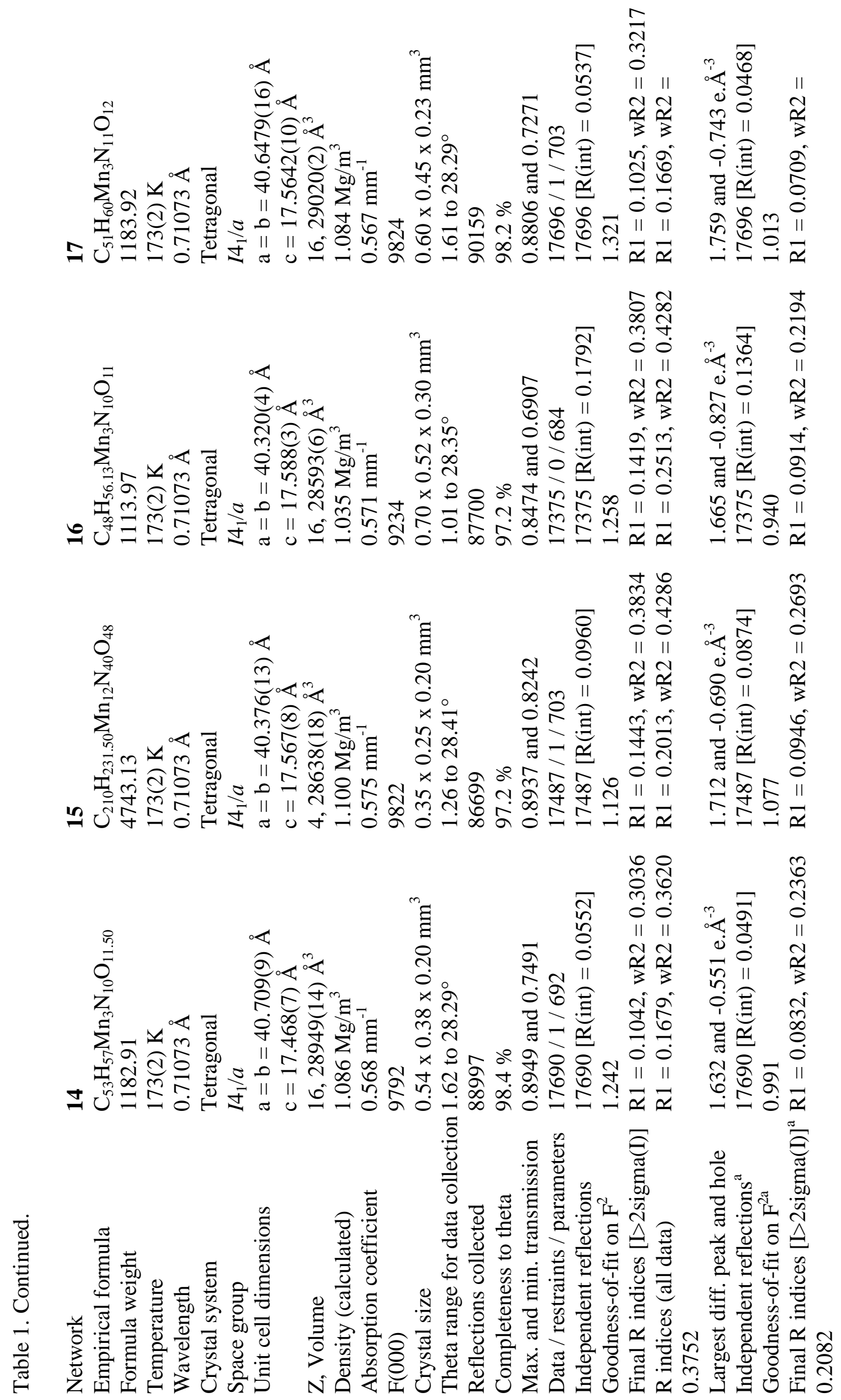


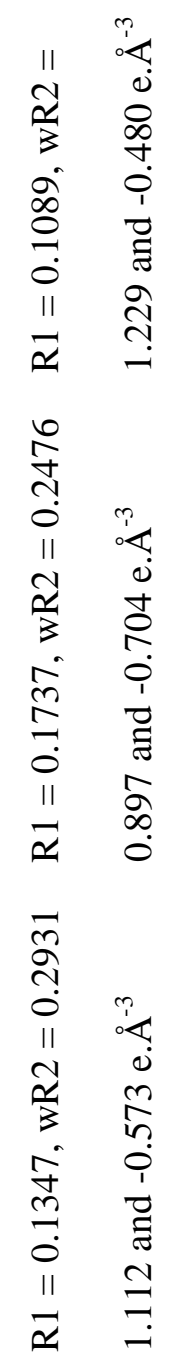

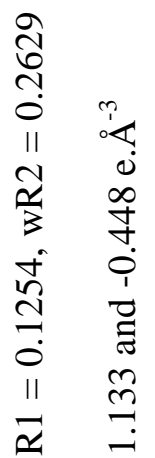

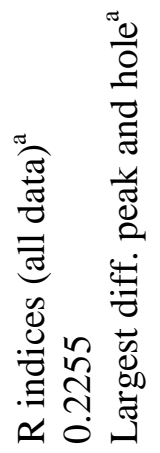



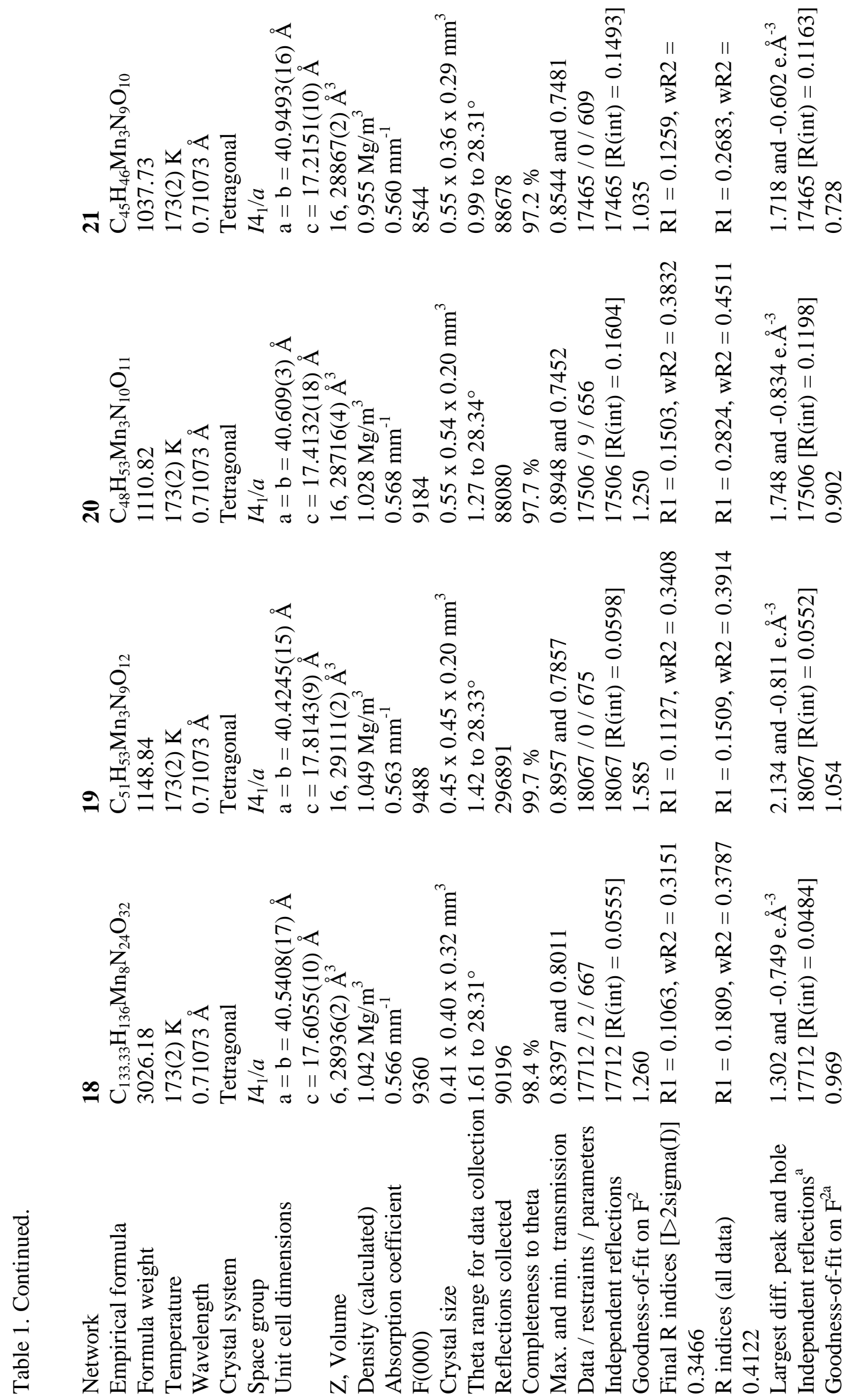


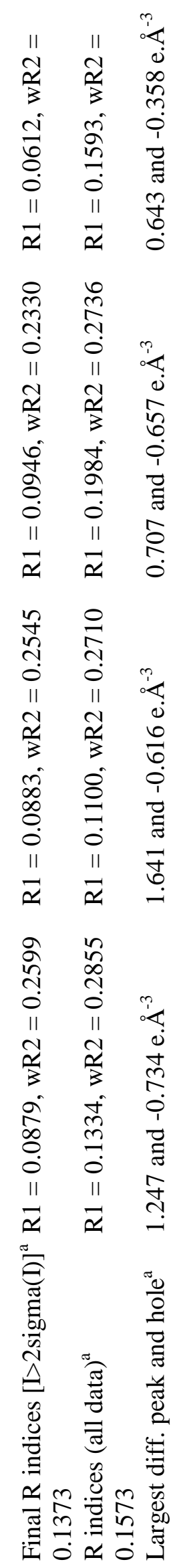




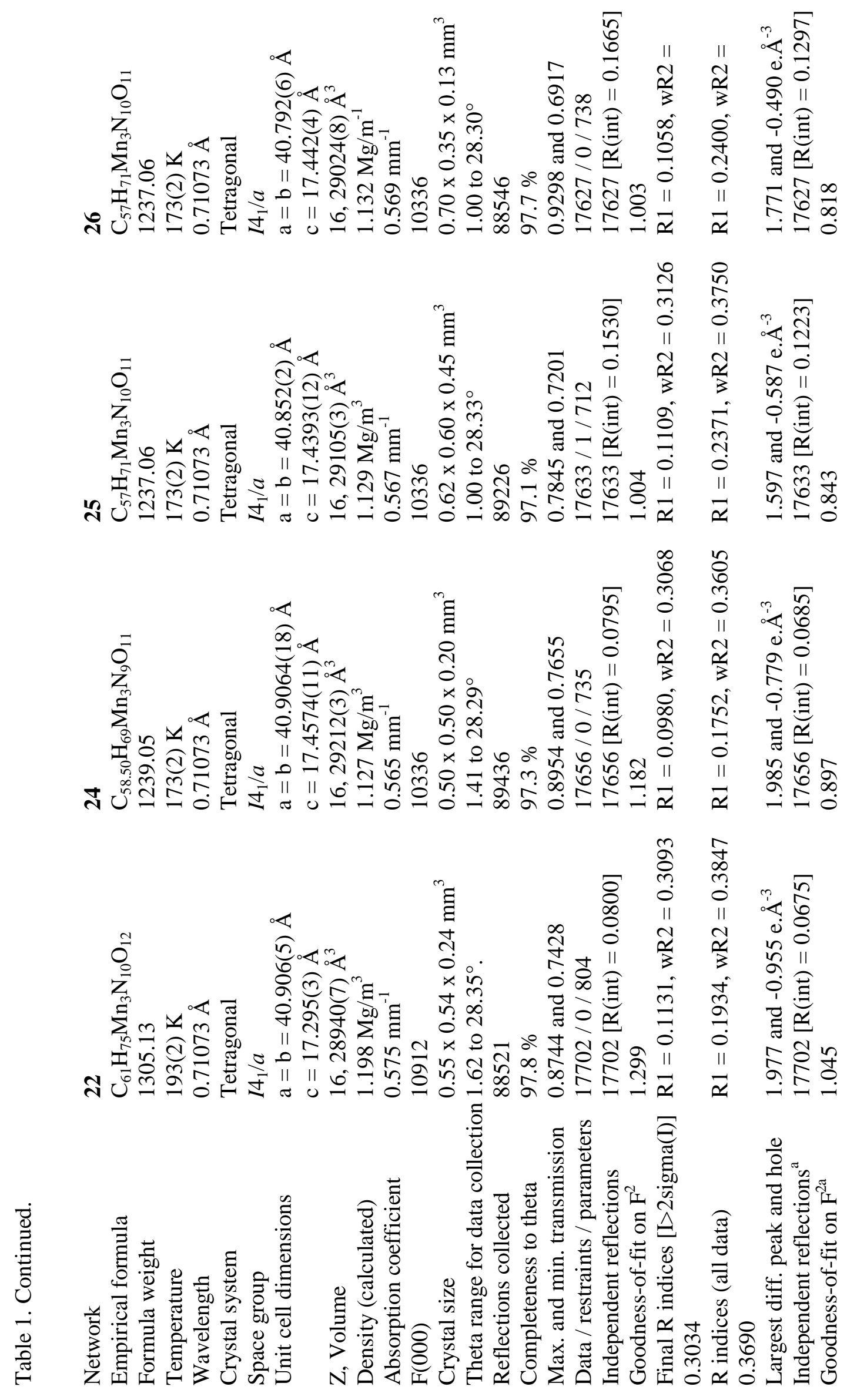




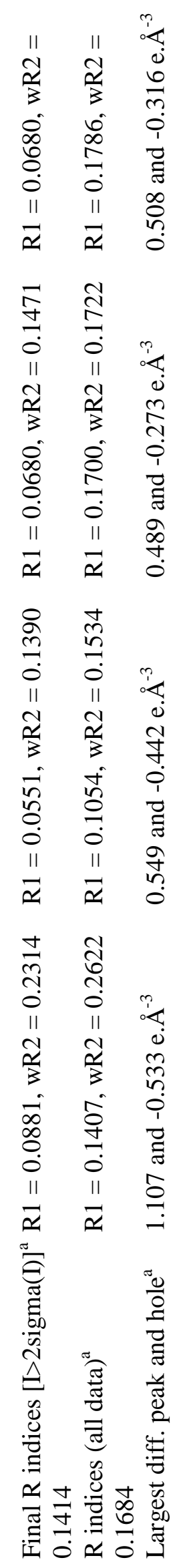



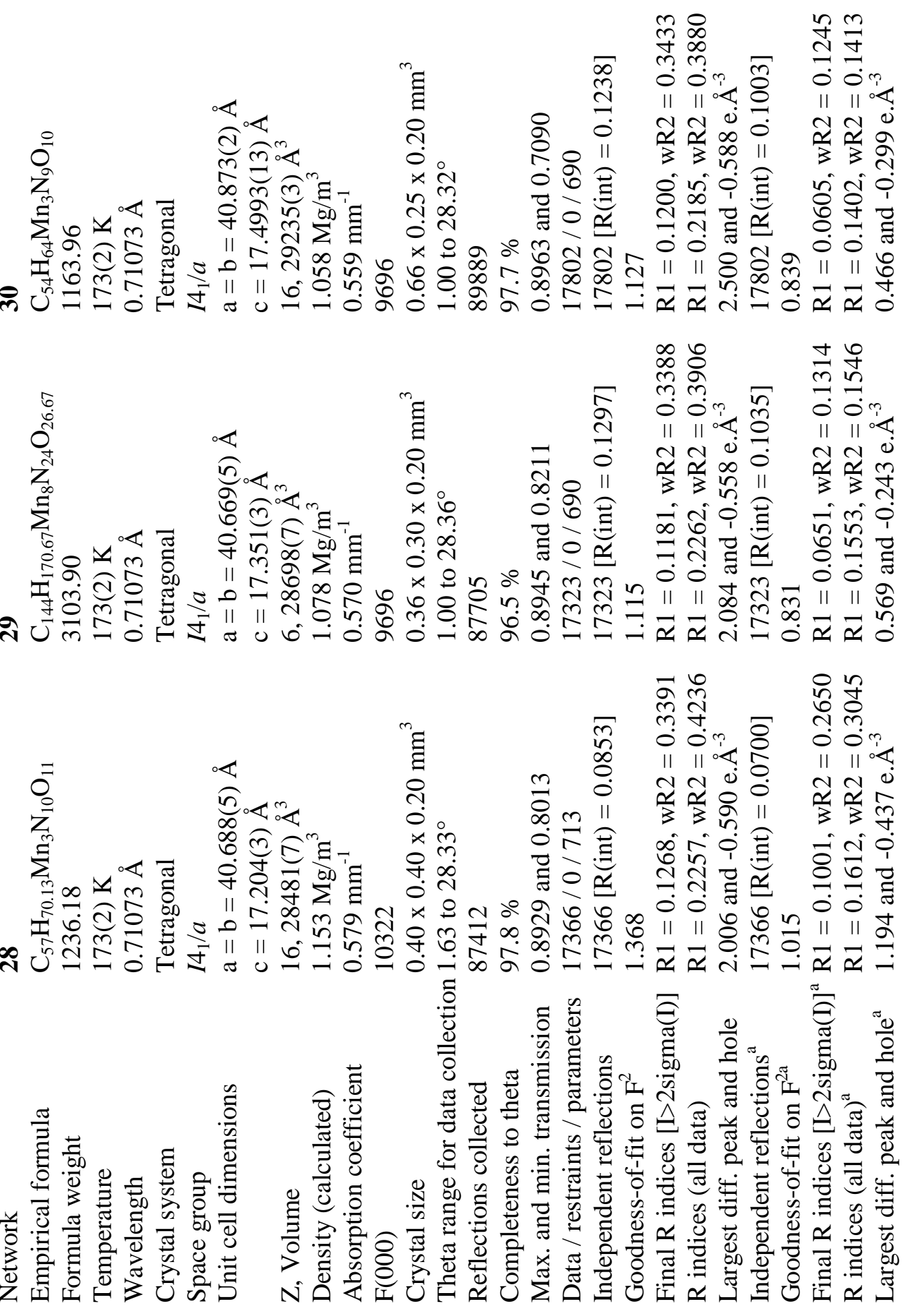


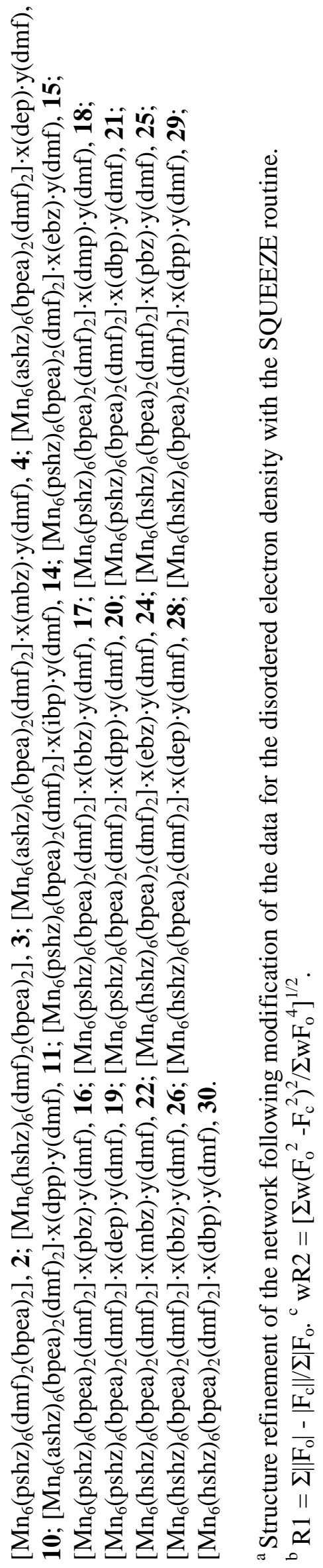

